\title{
PATTERN OF CARDIOVASCULAR AND PULMONARY LESIONS SEEN IN AN AUTOPSY SERIES OF SUDDEN AND UNEXPECTED DEATHS
}

\author{
Jini Lonappan Valooran ${ }^{1}$, Togy Thomas Zacharias ${ }^{2}$, Joy Augastine ${ }^{3}$, Anand T. P4, Rajendra Prasad 5 \\ ${ }^{1}$ Assistant Professor, Department of Pathology, Government Medical College, Thrissur, Kerala. \\ ${ }^{2}$ Assistant Professor, Department of Pathology, Government Medical College, Thrissur, Kerala. \\ 3 Professor and HOD, Department of Pathology, Amala Institute of Medical Sciences (AIMS), Thrissur, Kerala, India. \\ ${ }^{4}$ Assistant Professor, Department of Forensic Medicine, Government Medical College, Thrissur, Kerala. \\ ${ }^{5}$ Assistant Professor, Department of Forensic Medicine, Government Medical College, Thrissur, Kerala.
}

\section{ABSTRACT}

\section{BACKGROUND}

Cardiovascular diseases continue to be the most common generic cause of sudden and unexpected death. Atherosclerotic coronary heart disease and acute myocardial infarction are the most prevalent forms of fatal cardiac disease observed at autopsy. Other cardiac lesions and pulmonary lesions are frequently listed as a cause of death, but the prevalence of such lesions as the cause of death and incidental findings in the general population is unknown.

\section{MATERIALS AND METHODS}

Observational study of 500 forensic autopsies which were evaluated for cardiovascular and pulmonary pathologies.

\section{RESULTS}

The most frequently observed major finding was coronary artery disease. Of the 500 cases, cardiac disease was noted in 143 cases, pulmonary disease was in 79 cases and other vascular disease in 5 cases. Cardiovascular and pulmonary diseases constitute the major proportion of mortality in unexpected deaths.

\section{CONCLUSION}

The pattern of mortality shown in this study provides a fair idea of the burden of cardiovascular and pulmonary pathologies in Thrissur district of Kerala, although it is not a population-based data. Cardiovascular and pulmonary pathologies constitute the major share of mortality in unexpected deaths.

\section{KEYWORDS}

Sudden and Unexpected Deaths, Autopsy, Cardiovascular Pathology, Pulmonary Pathology.

HOW TO CITE THIS ARTICLE: Valooran JL, Zacharias TT, Augastine J, et al. Pattern of cardiovascular and pulmonary lesions seen in an autopsy series of sudden and unexpected deaths. J. Evolution Med. Dent. Sci. 2017;6(38):3069-3072, DOI: $10.14260 /$ Jemds/2017/662

\section{BACKGROUND}

An autopsy is a medical procedure that consists of a thorough examination performed on a body after death, to evaluate disease or injury that may be present and to determine the cause and manner of a person's death. An autopsy may be required in deaths that may have medical and legal issues. Causes of unnatural and sudden death are many, with accidents being the most common. The global burden of cardiovascular disease (CVD) has been increasing over time. At the beginning of the 20th century, CVD accounted for less than 10 percent of all documented deaths worldwide. At the start of the 21st century, it was the attributable factor in 30 percent of all deaths globally with more than 80 percent of those occurring in low and middle income countries. ${ }^{1}$

Financial or Other, Competing Interest: None.

Submission 25-03-2017, Peer Review 29-04-2017,

Acceptance 05-05-2017, Published 11-05-2017.

Corresponding Author:

Dr. Jini Lonappan Valooran,

Assistant Professor,

Department of Pathology,

Government Medical College,

Thrissur-680596,

Mulangunnathu Kavu (P. O.),

Thrissur District, Kerala.

E-mail: jinivalooran77@gmail.com

DOI: $10.14260 /$ jemds $/ 2017 / 662$
Cardiovascular and pulmonary disease continues to be the most common generic causes of sudden and unexpected deaths. ${ }^{2}$ The study focuses on the frequency and pattern of cardiovascular and pulmonary disease as the cause in sudden and unexpected deaths. We selected these two systems because they are most common attributable factors in sudden and unexpected deaths. ${ }^{2}$

\section{Objectives}

To assess the pattern of cardiovascular and pulmonary pathologies in unexpected deaths where cause of death was not clear and the age and sex distribution of various lesions in the above setting.

\section{MATERIALS AND METHODS}

The study was conducted in the Department of Pathology, Government Medical College, Thrissur, Kerala. It is a retrospective study of medicolegal autopsies that were sent to the Department of Pathology from the Department of Forensic Medicine, Government Medical College, Thrissur, Kerala. All cases had undergone a complete autopsy examination. Immediate cause of death was defined as the final disease or complication directly causing death. The contributing cause of death was defined as other significant conditions or disease that contributed to death but was not the immediate cause of death. 
The Cases have been sent for Pathological Examination from Department of Forensic Medicine for-

1. Confirmation of the diagnosis made at autopsy.

2. For identification of the cause of death when there was no definite conclusion at autopsy. In the present study, these cases were analysed with respect to the cardiovascular and pulmonary pathologies.

Gross examination of the specimens were done and tissue samples were taken from relevant areas and paraffin blocks made. 5 micron thin sections were cut and were stained with H\&E. The heart, great vessels and lungs were examined in all cases. Special stains (Reticulin, Masson Trichrome, EVG, GMS stain for Fungi and AFB) were done wherever required.

\section{Duration of the Study}

March 2007 to Feb 2013 (6 Years).

\section{Sample Size}

500 cases.

\section{Inclusion Criteria and Study Design}

Medicolegal autopsies conducted in the Department of Forensic Medicine, Government Medical College, Thrissur, Kerala, were selected. All cases with a known cause of death like RTA (except when cause of death was not obvious), burns, poisoning, blast injury, assault, fall from height were excluded.

\section{RESULTS}

During a period of 5 years and 8 months from March 2007 to November 2012, a total of 500 specimens of autopsies were studied.

\section{Cardiac Pathology}

$22.4 \%$ (112 cases) were found to have coronary artery disease. Age of cases ranged from 21 years to 95 years of age. Maximum number of CAD cases was in the sixth decade (28 cases) and fifth decade (27 cases). Male to female ratio of CAD was 12:1 in this series. Females were mostly affected in the fourth decade ( 4 cases) and fifth decade ( 3 cases).

Incidence of myocarditis was 3\%, distributed over an age distribution of first to sixth decade with an almost equal sex predilection (8 males and 7 females).

Hypertrophic cardiomyopathy was found in 6 cases all of whom were males and all of them were in the age group $3^{\text {rd }}$ and $4^{\text {th }}$ decade.

Valve pathology were of myxoid degeneration (2 cases of MVP) and of rheumatic nature (3 cases affecting both mitral and aortic valves) excluding the single case of infective endocarditis of mitral valve which was neither rheumatic nor myxoid degeneration.

There was one case each of ARVD, EMF and Neuroendocrine carcinoma. The neuroendocrine carcinoma was in a 78-year-old female who had presented with sudden respiratory distress and the tumour was infiltrating the cardiac muscle. The case of ARVD was in a 36-year-old male with sudden death which showed extensive fibrofatty infiltration of the right ventricular wall.

\section{Pulmonary Pathology}

The maximum number of pulmonary pathology was from lobar pneumonia (5.4\%) and was seen over 20 to 80 years of age. Next common finding was pulmonary tuberculosis
(4.2\%) all of which had characteristic caseation with necrosis. Among the Tuberculosis cases, six were part of miliary tuberculosis and one was a known case with HIV positive status.

There were 8 cases of interstitial pneumonia and 6 cases of bronchopneumonia and one case of fungal pneumonia. CVC lung was seen in 6 cases.

There were 2 cases of epiglottic abscess; both were young males (27 and 31 years old) who were labourers from northern part of India who had come to Kerala for employment (manual labourers).

5 cases, 3 males and 2 females had shown extensive emphysematous changes and 1 case, a 38-year-old male had typical histological features of ARDS. 2 cases had features of Status asthmaticus, one a 30-year-old male and the other an 18-year-old female.

Vascular pathologies, PPHN, Dissection of aorta, pulmonary thromboembolism, Fat and bone marrow embolism and Amniotic fluid embolism were present in one case each constituting a total of 5 . Dissection was in a 76year-old female who was found dead and had no preceding history of any significant illnesses. Fat and bone marrow embolism was found in a 26-year-old male RTA victim who died 3 days after the RTA. Pulmonary thromboembolism was seen in a 32-year-old female without any relevant past history.

\begin{tabular}{|c|c|}
\hline Coronary artery disease & 112 \\
\hline Myocardial bridging & 1 \\
\hline Myocarditis & 15 \\
\hline Infective endocarditis & 1 \\
\hline HCM & 6 \\
\hline EMF & 1 \\
\hline Neuroendocrine Tumour & 1 \\
\hline Valvular heart disease & 5 \\
\hline ARVD & 1 \\
\hline \multicolumn{2}{|c|}{ Total } \\
Table 1. Cardiac Pathology Frequency
\end{tabular}

\begin{tabular}{|c|c|c|c|}
\hline Age & Male & Female & Total \\
\hline $21-30$ & 13 & 1 & 14 \\
\hline $31-40$ & 17 & 4 & 21 \\
\hline $41-50$ & 27 & 3 & 30 \\
\hline $51-60$ & 28 & 1 & 29 \\
\hline $61-70$ & 10 & 3 & 13 \\
\hline $71-80$ & 4 & 0 & 4 \\
\hline $81-90$ & 1 & 0 & 1 \\
\hline $91-100$ & 0 & 0 & 0 \\
\hline Total & $\mathbf{1 0 0}$ & $\mathbf{1 2}$ & $\mathbf{1 1 2}$ \\
\hline \multicolumn{4}{|c|}{ Table 2. Age Distribution of CAD } \\
\hline
\end{tabular}

\begin{tabular}{|c|c|}
\hline ARDS & 1 \\
\hline Emphysema & 5 \\
\hline Lobar pneumonia & 27 \\
\hline Bronchopneumonia & 6 \\
\hline Fungal pneumonia & 1 \\
\hline CVC Lung & 6 \\
\hline Interstitial pneumonia & 8 \\
\hline Bronchial Asthma & 2 \\
\hline Tuberculosis & 21 \\
\hline Epiglottic abscess & 2 \\
\hline Total & 79 \\
\hline
\end{tabular}




\begin{tabular}{|c|l|}
\hline Primary pulmonary hypertension & 1 \\
\hline Dissection or Aorta & 1 \\
\hline Embolism (Fat and Bone marrow) & 1 \\
\hline Amniotic fluid embolism & 1 \\
\hline Pulmonary thromboembolism & 1 \\
\hline Total & $\mathbf{5}$ \\
\hline Table 4. Vascular Pathology \\
\hline
\end{tabular}

\begin{tabular}{|c|c|c|c|}
\hline Age & Males & Females & Total \\
\hline $21-30$ & 2 & 0 & 2 \\
\hline $31-40$ & 5 & 1 & 6 \\
\hline $41-50$ & 9 & 0 & 9 \\
\hline $51-60$ & 3 & 1 & 4 \\
\hline $61-70$ & 3 & 0 & 3 \\
\hline $71-80$ & 1 & 0 & 1 \\
\hline $81-90$ & 1 & 1 & 2 \\
\hline $91-100$ & 0 & 0 & 0 \\
\hline Total & $\mathbf{2 4}$ & $\mathbf{3}$ & $\mathbf{2 7}$ \\
\hline Table 5. Age and Sex Distribution of Lobar Pneumonias \\
\hline
\end{tabular}

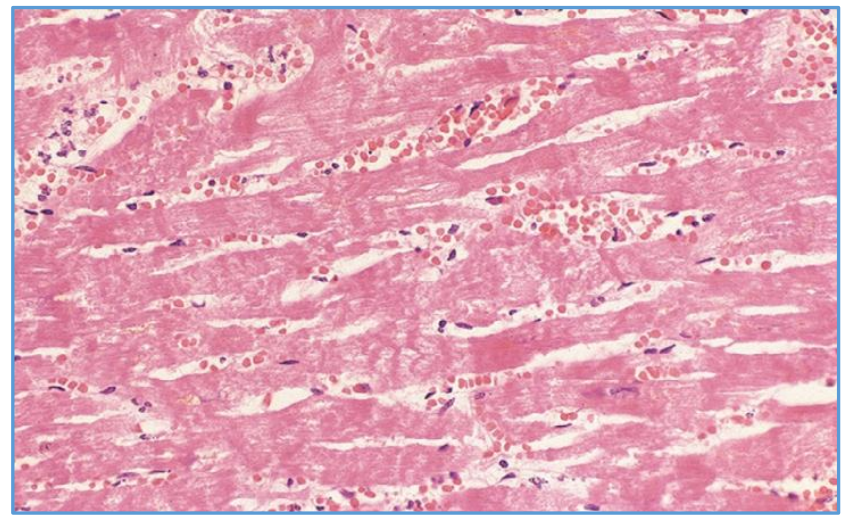

\section{Figure 3. Case of Myocardial Infarction Showing Cardiac Muscle with Coagulative Necrosis}

\section{DISCUSSION}

The goal of this study was to ascertain the frequency of various cardiovascular and pulmonary pathologies at autopsy where a definite cause of death was not clear or the findings at autopsy required histopathology confirmation. From India, very few studies have been conducted in this area. We looked specifically the cardiovascular and pulmonary pathologies, because they are the systems frequently associated with mortality.

Atherosclerotic coronary heart disease and myocardial infarction are the most frequent pathologies noted at autopsy. Other cardiovascular lesions and also pulmonary lesions are frequently listed as cause of death, but their prevalence in the general population is unknown.

In this study, 500 autopsy cases were studied where a definite cause of death was unclear during autopsy or required histopathology confirmation. Of the 500 cases, various cardiac disease were observed in 143 cases, pulmonary disease in 79 cases and vascular disease in 5 cases.

Catellier $\mathrm{MJ}^{2}$ et al have reported that the incidence of atherosclerotic coronary heart disease was the most common major finding observed in $16 \%$ cases. In the present study, the observed frequency of coronary artery disease was $22.4 \%$. (112/500).

Shirani $\mathrm{J}^{3}$ and colleagues reported an incidence of cardiac death in octogenarians of $53 \%$ and the incidence of coronary artery disease was $34.7 \%$. The higher incidence in their series is likely due to the higher age group selected.

Shirani $\mathrm{J}^{3}$ and colleagues reported the incidence of vascular disease as $13 \%$. In contrast the frequency in the present study was low (1\%). In an autopsy series published by Waller $\mathrm{BF}^{4}$ and colleagues, the incidence of CAD was $18 \%$. They observed 5\% incidence of MVP in contrast to a lower incidence of 0.4 in our series. Contrary to a high $24 \%$ incidence of myocardial bridging in their study, we just had one case of similar pathology.

The fact that rheumatic heart disease is progressively coming down in the society is evidenced by the markedly low incidence of valvular heart disease in our study. All cases of HCM had no other pathology in any other organs indicating the likely cause of death, arrhythmia. Myocarditis accounted for up to $44 \%$ of fatal events in major series on cardiac sudden death in the young. ${ }^{5}$ 
Pulmonary diseases were present in $15.8 \%$ of total cases of which the maximum share was of Lobar pneumonia (34.2\%) followed by Tuberculosis (26.5\%). Other pulmonary pathologies constituted minor shares including Emphysema. In our study, emphysema was present in 5 of the 500 cases (1\% of total cases.) This is in sharp contrast to other series published. (Tariq Mahmood Tahir and colleagues, 40\%, and Latif and coworkers, 6 43\% cases). Tariq et al reported $19.0 \%$ incidence of tuberculosis, whereas in our study the incidence of tuberculosis was $4.2 \%$. Male preponderance of tuberculosis is reported in many autopsy series. Norboo et al and Tariq et al reported cases of silicosis in their series, but we didn't have even a single case in our series. ${ }^{7}$ This is attributable to the geographical difference and the occupation of the people involved in the area. Niazi found 22 cases of pneumonia $(17.88 \%)$ in her study of pulmonary embolism in a total of 123 medico-legal autopsy cases. Corresponding figure in our series is $8.4 \%$ lobar pneumonia, interstitial pneumonia, bronchopneumonia and fungal pneumonia put together. The higher incidence of pneumonia in her study could be due to the reason that her study included $30 \%$ hospitalised patients who remained in the hospital for a longer or shorter period of time.

Various vascular pathologies was noticed in $13 \%$ of cases in an autopsy study of octogenarians by Shirani J and colleagues. ${ }^{3}$ We observed various vascular pathologies in only $1 \%$ of cases. This could be due to the inclusion of cases of extreme age in their series. Regarding pulmonary thromboembolism we came across just one in the entire series. This incidence is in contrast with previously reported studies of Sandler et $\mathrm{al}^{8}$ and Pineda et $\mathrm{al}^{9}$ which was found to be $10 \%$ and $9.1 \%$ respectively. This difference in incidence of PE could be the result of variability in the threshold for autopsy in cases of unconfirmed antemortem diagnosis.

This study has several limitations: the single centre design and obvious selection bias limit generalisability. Because this is a retrospective study, some specific data are lacking regarding events such as complete profile of risk factors of cardiovascular and pulmonary diseases, description of the clinical manifestations presented by victims before death or autopsy protocols. However, the study was performed in an academic tertiary care centre in which practices should be on par with other large hospitals. Additionally, other studies at similar institutions are available for comparison.

\section{CONCLUSION}

Cardiovascular and pulmonary pathologies constitute the major share of mortality in unexpected deaths. The patterns of mortality shown in this study may provide a fair idea of the burden of cardiovascular and pulmonary pathologies in Thrissur district of Kerala, although it is not a populationbased data. Therefore, to obtain a national pattern of CVD mortality there is a big need for further studies.

This may start with the collation of autopsy cases in all the major hospitals across the country, and subsequently the development of a population-based surveillance system. In the interim, specific studies may be conducted exploiting the demographic and surveillance systems that exist in the country.

\section{REFERENCES}

[1] Gaziano TA, Srinath KR, Fred P, et al. Disease control priorities in developing countries 2005:645-62.

[2] Catellier MJ, Waller BF, Clark MA, et al. Cardiac pathology in 470 consecutive forensic autopsies. J Forensic Sci 1990;35(5):1042-54.

[3] Shirani J, Yousefi J, Roberts WC. Major cardiac findings at necropsy in 366 American octogenarians. American Journal of cardiology 1995;75(2):151-6.

[4] Waller BF, Catellier MJ, Clark MA, et al. Cardiac pathology in 2007 consecutive forensic autopsies. Clin Cardiol 1992;15(10):760-5.

[5] Basso C, Calabrese F, Corrado D, et al. Myocarditis: an under- estimated cause of sudden cardiac death. In: Aliot E, Clementy J, Prystowsky EN, (eds). Fighting sudden cardiac death: a worldwide challenge, Armonk, NY: Futura 2000:447-58.

[6] Latif Z, Nagi AH. Emphysema in 100 cases: a postmortem study. Pakistan journal of pathology 1992;3:11-5.

[7] Norboo T, Angchuk PT, Yahya M, et al. Silicosis in a Himalaya village population: role of environmental dust. Thorax 1991;46(5):341-3.

[8] Sandler DA, Martin JF. Autopsy proven pulmonary embolism in hospital patients: are we detecting enough deep vein thrombosis? J R Soc Med 1989;82(4):203-5.

[9] Pineda LA, Hathwar VS, Grant BJ. Clinical suspicion of fatal pulmonary embolism. Chest 2001;120(3):791-5. 